\title{
Integration Colour and Texture Features for Content-based Image Retrieval
}

\author{
Hanan A. Al-Jubouri \\ Mustansiriyah University/ Department of Computer Engineering, Baghdad, Iraq \\ Email: hananaljubouri@uomustansiriyah.edu.iq
}

Received: 18 October 2019; Accepted: 12 January 2020; Published: 08 April 2020

\begin{abstract}
Content-Based Image Retrieval offers an automatic way to extract visual image contents such as colour, texture, and shape so-called extracted features. Due to growing volume of digital images, Content-Based Image Retrieval is emerged to store and retrieved images from large scale databases. However, Content-Based Image Retrieval faces a challenge of meaning "Semantic gap" between machine and human conceptual. How to reduce this gap between colour and/or texture features that represent an object in the image? It is still the challenge that basically related to the effectiveness of image representation by extracted features and similarity measures between a query image features and database image features. Hence, different visual features have been proposed such as Gary Level Co-occurrence Matrix (GLCM), Local Binary Pattern (LBP), and Discrete Wavelet Transform (DWT) texture features that are extracted from gray-scale images. This paper presents an unsupervised algorithm that exploits data and score-level fusion to address the semantic gap. The algorithm first extracts mentioned features from colour images in $H S V$ and $\mathrm{YCbCr}$ colour spaces to increase the effectiveness of image representation by integrating texture and colour visual information in terms of data-level fusion. Resulted similarity retrieval values are then fused in three versions of score-level fusion, summing values without weights, fixed, and adaptive weights using linear regression to raise relevant images in a ranked retrieved images list. WANG standard colour images are used to implement the algorithm. Rates of achievement in image retrievals are enhanced at both levels.
\end{abstract}

Index Terms - Content-Based Image Retrieval (CBIR), Gary Level Co-occurrence Matrix (GLCM), Local Binary Pattern (LBP), Discrete Wavelet Transform (DWT), data and score-level fusion.

\section{INTRODUCTION}

Nowadays, a massive of digital images being acquired and stored in databases in different areas of our life, social media, biometric, forensics, medical, education, etc.. Previously, image retrieval systems use keywords or phrases which are associated with images to represent and retrieve images from databases such as Yahoo. Due to limitations in such the way, Content-Based Image
Retrieval (CBIR) presents a flexible way to index images automatically based on visual content of images (i.e. colour, texture, and shape).

Images are represented in the database by extracting the visual content such as colour, texture, and/or shape feature vectors at off-line phase. Meanwhile, the system extracts a feature vector from a query image following the same procedure at on-line phase. A comparison between two extracted features vectors is made by using a similarity measure (i.e. distance function). The most similar images are ranked to the user as a retrieved list as shown in Fig. 1. However, CBIR systems are down front the semantic gap problem between a conceptual meaning and low-level visual features [1].

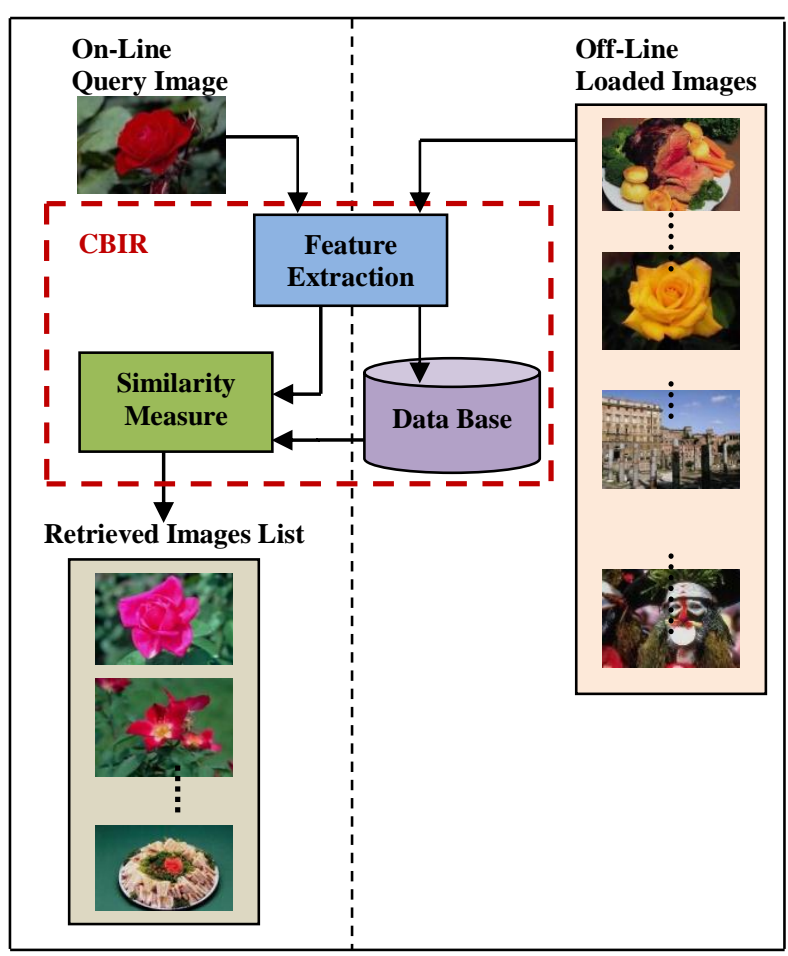

Fig.1. CBIR system

Consequently, interested researchers from different communities have been explored different features (global and local), similarity measurements, developed methods and algorithms [2] to reduce the challenge and the door is still open for more because image 
representation as low-level feature is a subjective to the human perceive in addition to the shortcoming of similarity measurements.

Hence, our motivations were firstly investigating global features where images are represented by a single vector means retrieval process faster than local features due to low dimension and computation, secondly, integrating texture and colour visual information in terms of data-level fusion to increase the effectiveness of GLCM, LBP, and DWT texture features, thirdly aggregating resulted similarity measurements from using mentioned features in terms of score-level fusion to raise average of relevant images in the ranked list. Therefore, an unsupervised algorithm was developed based on outcomes of above tests to address the challenge of CBIR. Section 2, 3, and 4 will explain LBP, GLCM, and DWT respectively. Section 5 will review related works. Section 6 will present the algorithm, experimental results and discussion. Finally, conclusions will be in Section 7.

\section{GRAY LEVEL CO-OCCURANCE MATRIX (GLCM)}

GLCM is a square matrix of intensity occurrences distribution. The matrix provides information about relative position of the neighbouring pixels (i.e. distance) in an image. Values of the matrix are the number of times that a greyscale intensity of pixel $i$ occurs with the adjacent value of pixel $j$. Because it is not invariant to rotation, four GLCM matrices are calculated for $0^{\circ}, 45^{\circ}$, $90^{\circ}$, and $135^{\circ}$ directions and then the average of them is taken. Fig. 2 shows co-occurrence matrix directions for a specific pixel where a distance is 1 neighbour pixel in four directions [3].

$90^{\circ}[-10]$

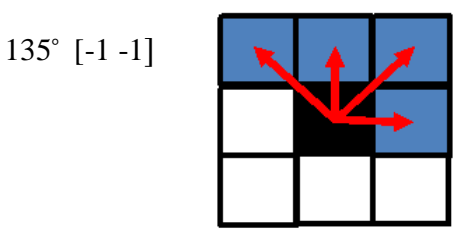

$45^{\circ}[-11]$

$0^{\circ}\left[\begin{array}{ll}0 & 1\end{array}\right]$

Fig.2. Four directions using 1 pixel distance.

Fig. 3 illustrates how GLCM is creating using 1 pixel distance in a symmetric horizontal adjacent pixel direction and 8 levels. For example, the number of times that the intensity value 4 adjacent to 2 is 2 . The occurrence of 1 to 1 intensity value is 2 times and so on until the matrix is filled.

Hence, Gray Level Co-occurance Matrix statistical method is used to capture texture of images. The texture bears important visual information to represent and index images in database. Different approaches have been developed using GLCM [4,5,6,7] with other features such as Local Binary Patterns and Discrete Wavelet Transform to increase the effectiveness of representing images. In other words, each feature can capture different visual information that are exploited together to reduce the semantic gap issue as will be explained in Sec. V. In this paper, mentioned three features are exploited to develop an algorithm in terms of data and score level fusion.

\begin{tabular}{|c|c|c|c|c|c|c|c|c|c|c|c|c|c|}
\hline & & & & & & 1 & 2 & 3 & 4 & 5 & 6 & 7 & 8 \\
\hline 4 & 2 & 3 & 5 & 8 & 1 & $\frac{2}{2}$ & 0 & 0 & 0 & 0 & 0 & 0 & 0 \\
\hline 1 & 1 & 6 & 7 & 4 & 2 & 0 & 0 & 2 & 2 & 0 & 0 & 0 & 0 \\
\hline 6 & 8 & 4 & 2 & 3 & 3 & 0 & 2 & 0 & 0 & 1 & 0 & 1 & 0 \\
\hline 7 & 4 & 5 & 1 & 1 & 4 & 0 & 2 & 0 & 0 & 1 & 0 & 2 & 1 \\
\hline & & & & & 5 & 1 & 0 & 1 & 1 & 0 & 0 & 0 & 1 \\
\hline & & & & & 6 & 0 & 0 & 0 & 0 & 0 & 0 & 1 & 1 \\
\hline & & & & & 7 & 0 & 0 & 0 & 2 & 0 & 0 & 0 & 0 \\
\hline & & & & & 8 & 0 & 0 & 0 & 1 & 1 & 0 & 0 & 0 \\
\hline
\end{tabular}

Fig.3. GLCM for (4 x 5) image.

\section{LOCAL BINARY PATTERN (LBP)}

LBP is also a measurement of a local texture using the relationships between a pixel and its neighbouring pixels, but in different way. The process starts by subtracting the central pixel value from the neighbouring pixel values. Then a binary number is used to refer to each corresponding result. Consequently, these binary numbers create the local binary pattern that are multiplied by weights of locations and summed to obtain the new value of the central pixel according to the following formula:

$$
\begin{gathered}
L B P_{N, R}\left(P_{c}\right)=\sum_{n=0}^{N-1} s\left(P_{n}-P_{c}\right) 2^{n} . \\
s(P)=\left\{\begin{array}{l}
1 \text { if } P \geq 0 \\
0 \text { if } P<0
\end{array}\right.
\end{gathered}
$$

where $N$ and $R$ are the number of neighbouring pixels and radius of neighbouring pixels. Fig. 4 illustrates above process for $3 \times 3$ block of the image.

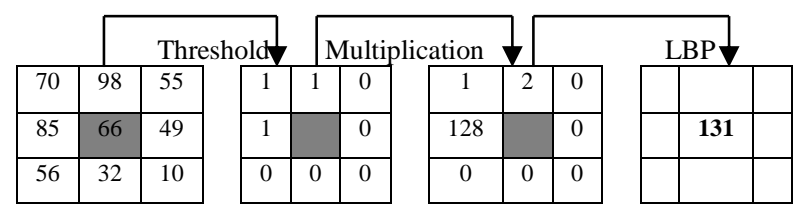

Fig.4. LBP for $N=8$ and $R=1$.

Suppose $\mathrm{A}$ is an image of $\mathrm{X}$ by $\mathrm{Y}$ dimension, the resulting $L B P_{N, R}$ code image can be represented by a histogram $h$ of length $K$, where $0 \leq k \leq K-1$ and $K=2^{N}$ is the number of all the LBP codes. For instance, if $N=8$ neighbours, then $K=256$. Although feature $h$ has good properties such as grey-scale invariance, low complexity, few parameters, and satisfactory discriminating power [22], the $h$ is a long histogram ( $2^{N}$ distinct values). Consequently, the study in [23] reported that not all of the local patterns are necessary to make texture analysis and proposed using just "uniform" patterns $L B P_{N, R}^{u 2}$. The uniform patterns contain at most two bitwise transitions from 0 to 1 or vice versa when the binary string is 
considered as circular 11000011. Uniform patterns consist of useful texture features compared to nonuniform binary patterns. Therefore, all occurrences of non-uniform patterns are aggregated to a single bin of the histogram. As a result, the number of bins in $h$ is reduced to 59-bins (i.e. 58 uniform patterns and 1 for non-uniform patterns).

\section{DiSCRET WAVELET TRANSFORM (DWT)}

DWT is a type of converting images from spatial domain to frequency domain where it can be analysed in multi resolutions or decomposition levels. There are many different wavelet filters such as Haar, Daubechies, and Coiflets used in practical applications.

In wavelet transform, a low-pass filter (L) and a highpass filter $(\mathrm{H})$ are two filters to use. Hence, the wavelet decomposition at level-one is four sub-bands (LL, HL, $\mathrm{LH}$, and $\mathrm{HH})$. In our experiments, we used three decomposition levels (Haar) as shown in Fig. 5.

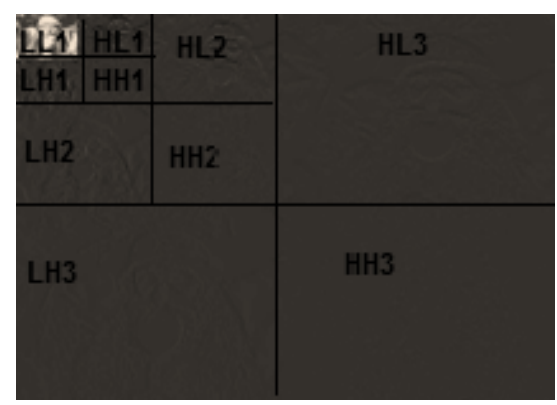

Fig.5. Wavelet decomposition.

\section{RELATED WORKS}

Different colour, texture, colour texture, and shape features have been explored and used to represent images effectively in CBIR. Prabhu and Kumar [4] used colour histogram, texture GLCM, and texture GLCM from Discrete Wavelet Transform (DWT) features to represent images in CBIR. Three databases of extracted features were built using WANG standard database images. Images were converted from RGB into HAV colour space and colour histogram features were calculated and quantized to 16-bin. Then K-means clustering algorithm was used to segment images that were indexed by centroids of clusters (F1) in the first database. GLCMs were created for images after HSV conversion to extract texture features (Energy, Contrast, Entropy, and Homegenity) (F2) in the second database. Images were converted into gray and DWT was calculated then GLCMs were created from low subband (LL) to represent images (F3) in the third database.

F1 was integrated to F2 and F3 separately in terms of data-level fusion to increase the effectiveness of image representation. Consequently, the performance of retrieval had been enhanced.
Bagri and Johari [5] used GLCM and Tamura texture features and Hu-moments shape feature to indexed images in the database. On one hand, the proposed retrieval method used GLCM texture and $\mathrm{Hu}$-moments shape features combination. On the other hand, the method used Tamura texture and Hu-moments shape features to reduce a semantic problem between low-level features and high conceptual meanings.

Benco et al. [6] investigated GLCM texture feature from grayscale images and CGLCM colour texture feature from $R G B$ and $H S V$ images by making some modification (i.e. 13 directions) on the original GLCM.

Puviarasan et al. [7] developed a CBIR system where RGB colour images were converted into grayscale. The system used a data-level feature that was obtained from integrating GLCM (Contrast, Energy, Entropy, Correlation, and Homogenity) texture and ( 7 moment invariant) shape features.

Methods in [8,9,10] exploited score-level fusion between different local and global features to reduce the semantic challenge in CBIR. Putri et al. [8] used HSV colour histogram and GLCM texture features that were extracted from segmented image and unsegmented image. That is means the features are local and global respectively. Lande et al. [9] used the same idea, where the local features were dominant colour and Fourier descriptors shape. Meanwhile, the global feature was GLCM. Kaur and Singh [10] used Center-Symmetric Local Binary Pattern (CSLBP), Local phase quantization (LPQ), and GLCM features.

Originally, LBP features used for face images by dividing images into blocks and histograms were concatenated to represent faces [11]. Then the using of LBP has been extended to represent natural scene. Recently, the feature integrated to the colour histogram for texture image retrieval and classification [12]. Meanwhile, the LBP feature was extracted from Hue channel for HSL images (LBPH), gray images (LBPC) and then combined or fused to the colour histogram. The aim is to enhance the accuracy of image retrieval [13].

\section{EXPERIMENTS}

This section clarifies a database that was used in conducted experiments, a framework of proposed algorithm that was followed, and retrieved image results were obtained using texture and colour-texture GLCM features at data and score-level fusion.

\section{A. Database}

WANG standard colour database images [16] which is part of Corel database and publically available for researchers. The standard database contains 1000 images in JPEG format that are divided into 10 categories (Elephants, Flowers, Buses, Foods, Horses, Mountains, African people, Beach, Buildings, and Dinosaurs). Each category has 100 images in (256x384) and (384 x 256) size. Fig. 6 shows sample of image categories. 


\section{B. The Evaluation of Performance}

A commonly measure to evaluate a proposed approach or system in terms of Content-based image retrieval is a precision, where the relevant images provide a baseline to make a computation.

$$
P=\frac{N R M}{T R M}
$$

where, $P$ is the precision of image retrieval, $N R M$ is number of relevant retrieved images and TRM is total number of retrieved images.

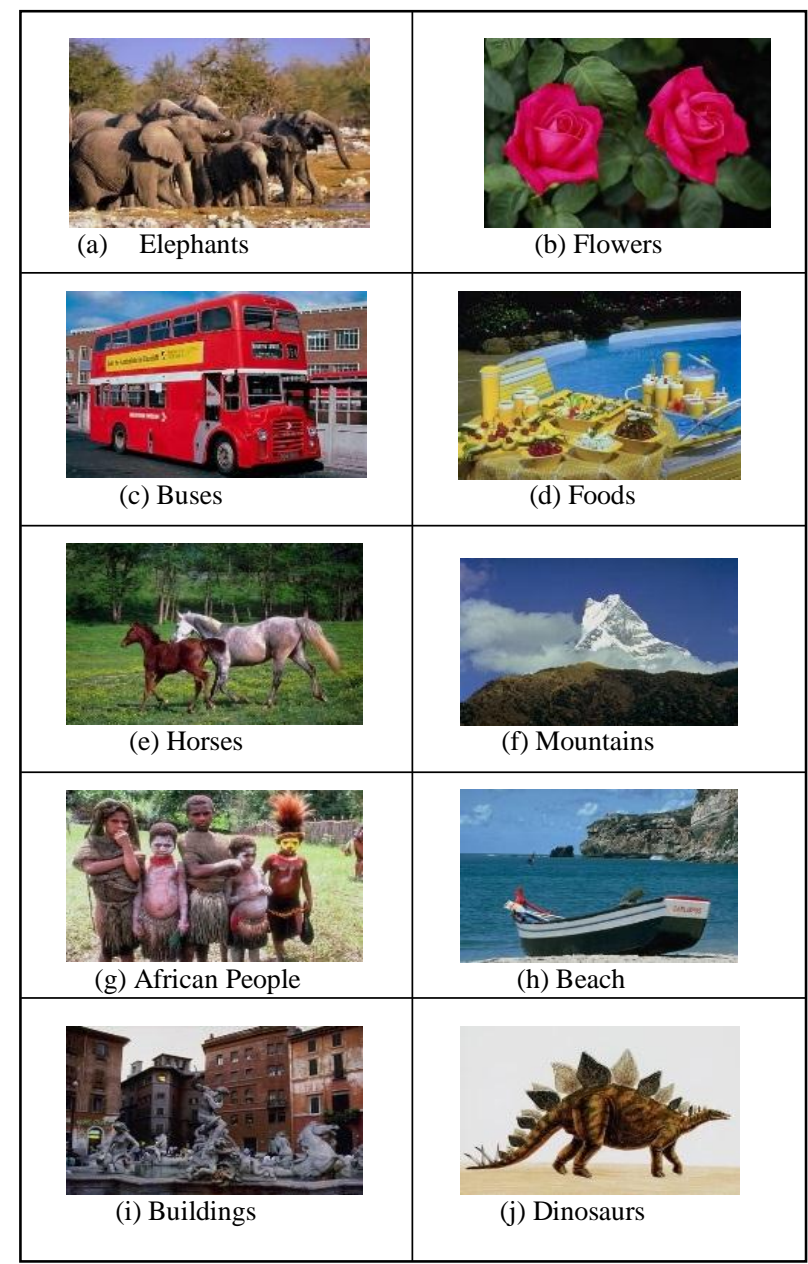

Fig.6. Sample of WANG database

$$
A P=\frac{\sum_{i=1}^{n} P_{i}}{n} .
$$

where, $A P$ is average precision of image retrieval, $P_{i}$ is precision of $i$ image in the class, and $n$ is total number of images in the class.

$$
M A P=\frac{\sum_{j=1}^{m} A P_{j}}{m}
$$

where, MAP is mean average precision of image retrieval, $A P_{j}$ is average precision of $j$ class image, and $m$ is total number of classes in the database.

\section{Framework}

Fig.7 shows stages of a proposed algorithm, preprocessing, feature extraction, data-level fusion, similarity measurement, and score-level fusion. The preprocessing stage is converting images spaces separately. GLCM, LBP, and DWT are calculated at the feature extraction stage. These features from $H, S$, and $V$ or from $Y, C b$, and $\mathrm{Cr}$ are integrated at data-level fusion stage. Resulted features are stores in the database images at offline phase. The same stages are followed for a query image at on-line phase. Obtained features are matched with those in the database using City block distance function at the similarity measurement stage. Resulted similarity values are normalized and then summed without using weights, fixed, and adaptive weights separately at the score-level fusion stage. Finally, values are ranked in ascending order to retrieve a list of images. More details will be explained in next sections.

\section{Experiments, Results and Analysis}

Two scenarios were followed to investigate GLCM, LBP, and DWT features when the features were extracted from gray-scale images and extracted from $H S V$ and $\mathrm{YCbCr}$ colour images. The reason behind that is to investigate the features effectiveness in terms of texture and colour-texture visual information.

\section{Data-Level Fusion}

Fusin is integrating or combing between two or more features and originally used in information retrieval. There are two types of fusions which are data and scorelevel fusion $[19,20]$. Both types were adapted to our work. In this section, we will focus on the first one.

\section{a. GLCM Colour-Texture Feature}

In the first experiment, GLCMs were calculated from gray images and then four texture features were extracted to represent images in the database. These features are Energy, Homogeneity, Contrast, and Correlation $\left(f_{1-4}\right)$ which are common in use. Because GLCM is not constant to rotation, four directions $\left(0^{\circ}, 45^{\circ}, 90^{\circ}\right.$, and $\left.135^{\circ}\right)$ were taken into account. In other words, four GLCM metrics were created and then mentioned texture features were calculated. In terms of offset which is distance from an interest pixel to neighbours, 1 to 4 distance pixels were tested.

$$
\begin{gathered}
f_{1}=\sum_{i=1}^{N} \sum_{j=1}^{N} p(i, j)^{2} . \\
f_{2}=\sum_{i=1}^{N} \sum_{j=1}^{N} \frac{p(i, j)}{1-|i-j|} . \\
f_{3}=\sum_{n=0}^{N-1} n^{2}\left\{\sum_{\substack{i=1 \\
|i-j|=n}}^{N} \sum_{j=1}^{N} p(i, j)\right\} .
\end{gathered}
$$

where $p(i, j)$ is a normalized value of a matrix GLCM and $N$ is a number of gray levels of GLCM. 


$$
f_{4}=\sum_{i=1}^{N} \sum_{j=1}^{N} \frac{\left(i-\mu_{i}\right)\left(i-\mu_{j}\right) p(i, j)}{\sigma_{i} \sigma_{j}}
$$

where $\mu_{i}, \mu_{j}, \sigma_{i}, \sigma_{j}$ are mean and standard deviation of $p_{i}$ and $p_{j}$.

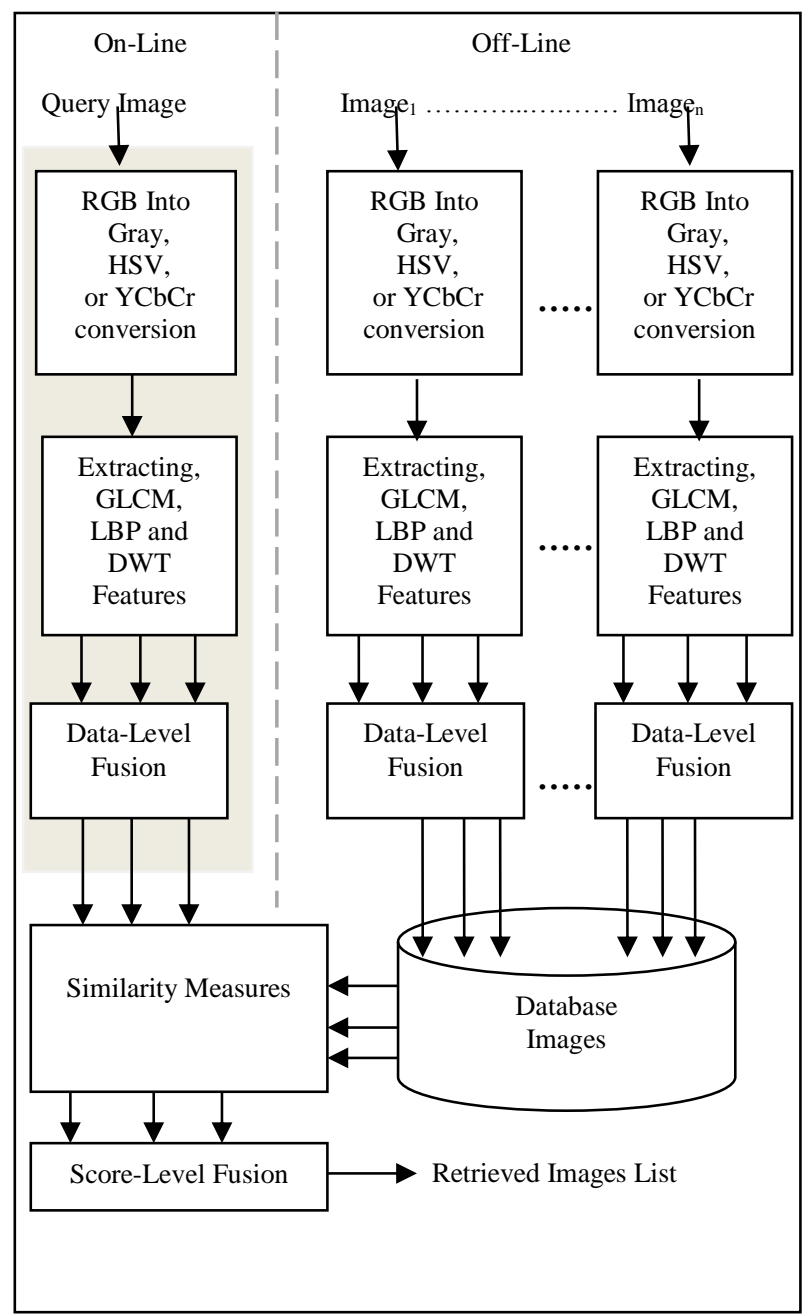

Fig.7. Framework of the proposed algorithm.

Table 1 illustrates MAPs of image retrieval for Top 1050 images using offset 1, 2, 3, and 4 pixels. As we can see that results are roughly close among four offsets but the best one is using distance 2 pixels. This led us to extend the investigation to colour texture GLCM feature rather than just texture as it has been done in existing work. However, we tested integration features in terms of data and score-level features as well as directed GLCM as will be showed later.

In the second experiment, the data-level fusion was implemented. Two colour spaces has been chosen from where the colour texture GLCM features were extracted, $H S V$ and $Y C b C r$. The $H S V$ colour space is abbreviation to Hue, Saturation, and Value channels where $V$ represents the brightness whereas $H$ and $S$ represent chromatic information. $H S V$ was developed to approximate colour realization and interpretation of human. Meanwhile, the $\mathrm{YCbCr}$ colour space is beneficial in compression such as video where more luminance information in $Y$ channel whereas blue and red colour information in $\mathrm{Cb}$ and $\mathrm{Cr}$ colour channel $[15,16]$.

The implementation of data-level fusion was done by concatenation Energy, Homogeneity, Contrast, and Correlation $\left(F_{H S V}\right)$ features from $H, S$, and $V$ channels to integrate colour and texture visual image content that is used to index images in the database.

Table 2 shows MAPs image retrieved results for 1, 2, 3, and 4 pixel distances (i.e. offset (1-4) pixels). It can be regard using 2 pixels distance can produce the best rates and achieve about (3-7) \% along Top 10 to 50 retrieved images. This means that colour information increases discrimination between images.

Table 3. illustrates more interest performance using $\mathrm{YCbCr}$ colour space with offset 4 about (1-2) \% higher than the performance $H S V$. This is evidence that colour information in $\mathrm{Cb}$ and $\mathrm{Cr}$ channels supported the recognition between classes of images. Hence, next investigation to the GLCM was made using the and $\mathrm{YCbCr}$.

In the third experiment, we observed that using values of GLCM directly to index images in the database has been succeed in face recognition for grayscale images [18] and noticed there is no implementation of the directed GLCM on natural colour images. Therefore, we used offset 4 to create directed GLCMs in four directions $\left(0^{\circ}\right.$, $45^{\circ}, 90^{\circ}$, and $\left.135^{\circ}\right)$ and calculate their averages for $Y, C b$, and $\mathrm{Cr}$ channels and concatenated them to represent natural scene colour images.

Resulted MAPs as long as Top 10-50 retrieved images were $(64,60,57,54$, and 52) \% respectively. This means directed GLCM satisfied 9\% higher than using Energy, Homogeneity, Contrast, and Correlation feature from GLCM (Table 3). Hence, using directed frequencies of GLCMs as feature $\left(\mathrm{DGLCM}_{Y C b C \mathrm{r}}\right)$ is more accurate and discriminates in representing natural scene colour images.

Table 1. MAPs for Top 10-50 Retrieved Images using GLCM Feature from Gray-Scale Images

\begin{tabular}{|c|c|c|c|c|}
\hline $\begin{array}{c}\text { Retrieved } \\
\text { Images }\end{array}$ & Pixel 1 & Pixel 2 & Pixel 3 & Pixel 4 \\
\hline Top 10 & 0.46 & 0.48 & 0.48 & 0.48 \\
\hline Top20 & 0.43 & 0.45 & 0.44 & 0.44 \\
\hline Top 30 & 0.41 & 0.42 & 0.42 & 0.41 \\
\hline Top 40 & 0.39 & 0.41 & 0.40 & 0.39 \\
\hline Top 50 & 0.38 & 0.39 & 0.38 & 0.38 \\
\hline
\end{tabular}

Table 2. MAPs for Top 10-50 Retrieved Images using GLCM Feature from HSV Images

\begin{tabular}{|c|c|c|c|c|}
\hline $\begin{array}{c}\text { Retrieved } \\
\text { Images }\end{array}$ & Pixel 1 & Pixel 2 & Pixel 3 & Pixel 4 \\
\hline Top 10 & 0.54 & 0.55 & 0.55 & 0.55 \\
\hline Top20 & 0.49 & 0.50 & 0.49 & 0.49 \\
\hline Top 30 & 0.46 & 0.46 & 0.46 & 0.45 \\
\hline Top 40 & 0.44 & 0.44 & 0.43 & 0.42 \\
\hline Top 50 & 0.42 & 0.42 & 0.41 & 0.40 \\
\hline
\end{tabular}


Table 3. MAPs for Top 10-50 Retrieved Images using extracted GLCM Feature from YCbCr Images

\begin{tabular}{|c|c|c|c|c|}
\hline $\begin{array}{c}\text { Retrieved } \\
\text { Images }\end{array}$ & Pixel 1 & Pixel 2 & Pixel 3 & Pixel 4 \\
\hline Top 10 & 0.51 & 0.54 & 0.54 & 0.55 \\
\hline Top20 & 0.47 & 0.50 & 0.50 & 0.51 \\
\hline Top 30 & 0.44 & 0.47 & 0.47 & 0.48 \\
\hline Top 40 & 0.43 & 0.45 & 0.45 & 0.45 \\
\hline Top 50 & 0.41 & 0.43 & 0.43 & 0.43 \\
\hline
\end{tabular}

We can make a conclusion based on above experiments. Integrating the colour and texture is more effective than the texture features using GLCM to represent images. Table 4 illustrates average precision for entire WANG database for Top 10 retrieved images to clarify and analysis the significance of the performance differences between both features.

Table 4. MAPs for Top 10 Retrieved Images using extracted GLCM features from YCbCr and Gray-Scale Images

\begin{tabular}{|c|c|c|}
\hline Classes & DGLCM $_{\text {YCbCr }}$ & Gray GLCM \\
\hline Elephants & 0.56 & 0.30 \\
\hline Flowers & 0.76 & 0.80 \\
\hline Buses & 0.69 & 0.70 \\
\hline Foods & 0.58 & 0.34 \\
\hline Horses & 0.92 & 0.57 \\
\hline Mountains & 0.46 & 0.22 \\
\hline People & 0.68 & 0.32 \\
\hline Beach & 0.38 & 0.34 \\
\hline Buildings & 0.42 & 0.28 \\
\hline Dinosaurs & 0.99 & 0.95 \\
\hline MAP & 0.64 & 0.48 \\
\hline
\end{tabular}

The static $t$-test method [24] was used to justify the significance.

$$
t=\frac{\overline{\mathrm{x}}-\bar{y}}{\sqrt{\frac{\mathrm{s}_{x}^{2}}{n}-\frac{\mathrm{s}_{y}^{2}}{m}}} .
$$

where $\bar{x}$ and $\bar{y}$ are the sample precision rates, $s_{x}$ and $s_{y}$ are the sample standard deviations, and $n$ and $m$ are the sample sizes. In our experiments, the size of the sample is ( $n=m=100$ precision values). The hypotheses are stated as follows. The null hypothesis $\left(\mathrm{H}_{0}\right)$ is that $\bar{x}-\bar{y}=0$. The alternative hypothesis $\left(\mathrm{H}_{\mathrm{A}}\right)$ is that $\bar{x}-\bar{y} \neq 0$.

$P$-value of the $t$-test is the probability of observing a test, where small value means the null hypothesis is not true.

The $t$-test was applied using MATLAB upon two samples of precision values GLCM texture and $F_{D G L C M}$ colour texture features to represent images. A returned value $\mathrm{H}=0$ refers to the acceptance of the null hypothesis, and a returned value $\mathrm{H}=1$ refers to a rejection of the null hypothesis.

Fig. 8 shows the hypothesis values returned against image classes in WANG databases. It is clear that performance differences are significant for 7 out of the performance differences are significant for 7 out of the total 10 classes, where $p$-values for Elephants, Foods, Horses, Mountains, People, Buildings, and Dinosaurs classes are 5.65E-16, 1.26E-08, 6.13E-19, 1.22E-12, $1.48 \mathrm{E}-19,2.71 \mathrm{E}-05$, and 0.003485 respectively. Hence, the visual colour information participate texture to increase the discrimination between images in different classes that could contain common objects.

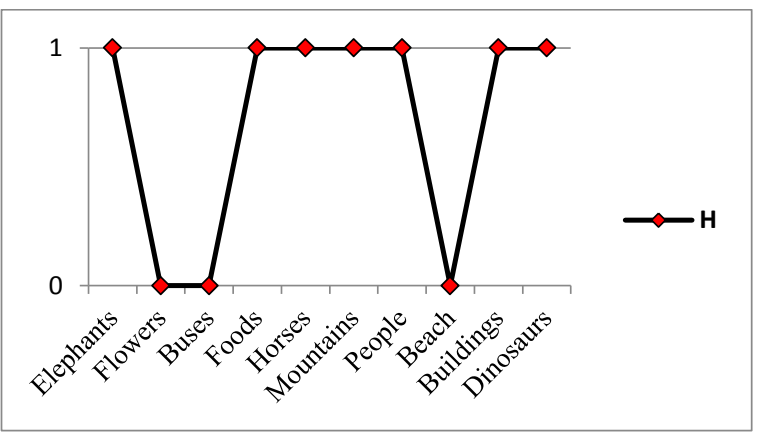

Fig.8. Hypothesis Values using GLCM.

\section{b. LBP Colour-Texture Feature}

In this experiment, $\mathrm{LBP}_{N, R}$ codes were calculated based on $N=8$ neighbours and $R=1$ radius. Firstly, LBP uniform histogram feature (59D) was extracted from gray-scale images $\left(\mathrm{LBP}_{\mathrm{GH}}\right)$ and secondly, the same feature was extracted from colour images in $\mathrm{YCbCr}$ colour space $\left(\mathrm{LBP}_{Y C b C r}\right)$. Therefore, $\mathrm{LBP}_{Y C b C r}(177 \mathrm{D})$ is a data-level feature because three uniform histograms from $Y, C b$, and $\mathrm{Cr}$ are concatenated. In addition, we explored that weighting the feature by $0.6,0.3$, and 0.1 respectively increased the effectiveness.

As we can see from Table 5, integrating colour and texture in $\mathrm{LBP}_{Y C b C r}$ raised the accuracy of retrieval about (13-10) $\%$ as long as Top10-50 retrieved images compared to $\mathrm{LBP}_{\mathrm{G}}$. Due to colour visual content affected on the representation of images positively.

Table 5. MAPs for Top 10-50 Retrieved Images using LBP Features from Gray-Scale and $\mathrm{YCbCr}$ Images

\begin{tabular}{|c|c|c|}
\hline Retrieved Images & LBP $_{\mathbf{G H}}$ & LBP $_{\text {YCbCrH }}$ \\
\hline Top 10 & 0.59 & 0.72 \\
\hline Top20 & 0.55 & 0.66 \\
\hline Top 30 & 0.52 & 0.63 \\
\hline Top 40 & 0.49 & 0.60 \\
\hline Top 50 & 0.47 & 0.57 \\
\hline
\end{tabular}

Table 6. APs for Top 10 Retrieved Images using extracted LBP features from $\mathrm{YCbCr}$ and Gray-Scale Images

\begin{tabular}{|c|c|c|c|}
\hline Classes & LBP $_{\text {YCbCr}}$ & LBP $_{\mathbf{G}}$ & $\boldsymbol{P}$-value \\
\hline Elephants & 0.60 & 0.34 & $8.46 \mathrm{E}-15$ \\
\hline Flowers & 0.91 & 0.87 & 0.167364 \\
\hline Buses & 0.97 & 0.94 & 0.03561 \\
\hline Foods & 0.75 & 0.44 & $1.53 \mathrm{E}-13$ \\
\hline Horses & 0.81 & 0.66 & $7.97 \mathrm{E}-05$ \\
\hline Mountains & 0.42 & 0.30 & $1.75 \mathrm{E}-05$ \\
\hline People & 0.67 & 0.55 & 0.008505 \\
\hline Beach & 0.49 & 0.46 & 0.516463 \\
\hline Buildings & 0.63 & 0.41 & $4.36 \mathrm{E}-07$ \\
\hline Dinosaurs & 0.98 & 0.97 & 0.470673 \\
\hline
\end{tabular}


We also used the $t$-test to make a justification between $\mathrm{LBP}_{\mathrm{G}}$ and $\mathrm{LBP}_{Y C b C r}$ for Top 10 retrieved images (Table 6). Consequently, Fig. 9 illustrates the hypothesis values that clarify the significance is happened with Elephants, Foods, Buses, Horses, Mountains, People, and Buildings. The probabilities of observing (i.e. $p$-values) for these classes are showed in last column of Table 6.

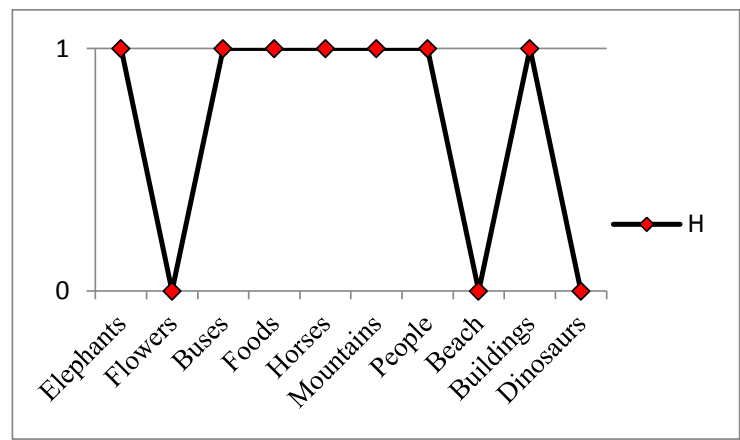

Fig.9. Hypothesis Values using LBP.

\section{c. DWT colour texture feature}

Here, the same scenario was followed to investigate Discrete Wavelet Transform Haar three sub-bands. On the one hand, statistical measures mean and standard deviation were calculated from gray-scale images as $\mathrm{DWT}_{\mathrm{GHaar}}$ feature (20D). On the other hand, the measures were extracted from $\mathrm{YCbCr}$ images as $\mathrm{DWT}_{Y C b C r}$ feature (60D). There were a high promotion of image retrieval performance about (15-21) \% using data-level feature $\left(\mathrm{DWT}_{Y C b C r}\right)$ as shown in Table 7.

Table 7. MAPs for Top 10-50 Retrieved Images using DWT Features from Gray-Scale and YCbCr Images

\begin{tabular}{|c|c|c|}
\hline $\begin{array}{c}\text { Retrieved } \\
\text { Images }\end{array}$ & DWT $_{\text {YCbCr }}$ & DWT $_{\mathbf{G}}$ \\
\hline Top 10 & 0.59 & 0.37 \\
\hline Top20 & 0.54 & 0.35 \\
\hline Top 30 & 0.51 & 0.33 \\
\hline Top 40 & 0.49 & 0.32 \\
\hline Top 50 & 0.47 & 0.32 \\
\hline
\end{tabular}

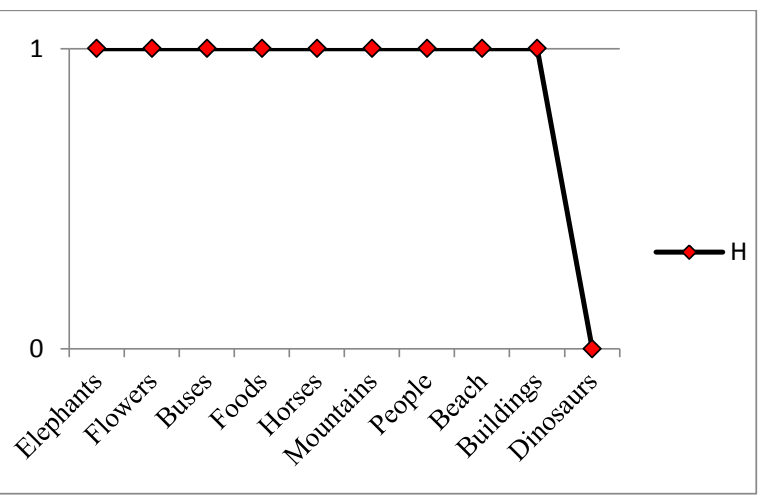

Fig.10. Hypothesis Values using DWT.

In terms of $t$-test for Top 10 retrieved images using above two DWT features, Fig. 10 illustrates the significant improvements appear with whole image classes except Dinosaurs class. This means that colour information participated to decrease the confusion between image classes even the feature in frequency domain.

To sum up, outcomes of above experiments using different features (GLCM, LBP, and DWT) in terms of data-level fusion contributed to narrow the semantic gap by integrating colour and texture visual information in image representation.

\section{Score-Level Fusion}

Score-level is another type of fusion that combines two or more different scores. We decided to use this type of fusion in addition to the data-level fusion. Therefore, we developed an algorithm that involves both fusion types to address the semantic gap issue as illustrated in Fig. 2 .

In this experiment, we suggested combining scores (i.e. similarity measures) obtained from $\mathrm{LBP}_{Y C b C r}$ DGLCM $_{Y C b C r}$, and $\mathrm{DWT}_{Y C b C r}$ data-level features using three versions of the score-level fusion. The first version is summing scores without using weights. The second version is summing scores with fixed weights $\left(\left(\mathrm{LBP}_{Y C b C r}\right.\right.$, $\left.\mathrm{w}_{1}=0.6\right), \quad\left(\mathrm{DGLCM}_{Y C b C r}, \quad \mathrm{w}_{2}=0.3\right), \quad$ and $\quad\left(\mathrm{DWT}_{Y C b C r}\right.$, $\left.\mathrm{w}_{3}=0.1\right)$ ). The third version is summing scores using a linear regression to determine weights automatically as our previous work [17].

The linear regression used to find the best fit straight line through points. This line is called a regression line which minimize the sum of squared errors of the estimation

To clarify the idea of a straight line as in (10) where $P$ is input matrix, $q$ is output and $x$ (coefficients) is the demand. a straight line equation was adapted to estimate weights, where $P$ represents dissimilarity values, $x$ are weights, and $q$ is the fusion vector.

$$
P x+q=0 \quad P x=q .
$$

where $P$ is input matrix $q$ is output and $x$ (coefficients) is the demand.

Suppose $x$ and $y$ dissimilarity values based on two different extracted features and $\bar{x}$ and $\bar{y}$ are means, then the best line model $(q)$ can be computed by:

$$
\begin{gathered}
q=\text { slope } * x+\text { intercept } \\
\text { slope }=\frac{n \sum x y-\sum x \sum y}{n \sum x^{2}-\left(\sum x\right)^{2}} \\
\text { intercept }=\bar{y}-\text { slope } * \bar{x}
\end{gathered}
$$

Now, we can find $w$ weights from the following a multiple linear regression instruction in MATLAB: $x=$ regress $(q, P)$ and then use them in the fusion formula as follows:

$$
\begin{gathered}
f_{i}=x \times w+y \times(1-w) \\
f_{i+1}=f_{i} \times w+z \times(1-w)
\end{gathered}
$$


where $=1 . . n-1, n$ is number of fused features, $x, y$, and $z$ are normalized scores.

At the last step, the final $f$ scores are sorted in ascending order to retrieve images

Outcomes of the proposed algorithm for the fusion versions are clarified in Fig. 11. The best algorithm performance is using a fixed weights fusion. Meanwhile, a regression version can determine weights automatically and satisfy performance like a sum version without weights. But it could be useful with other different features.

Hence, the algorithm contributes towards reducing the semantic issue in CBIR by using different data-level fusion features from spatial and frequency domain and then aggregating normalized scores which are obtained from these features in terms score-level fusion. The highest accuracy of retrieval is $79 \%$ at Top 10 retrieved images that compared to other methods from literature in Table 8.

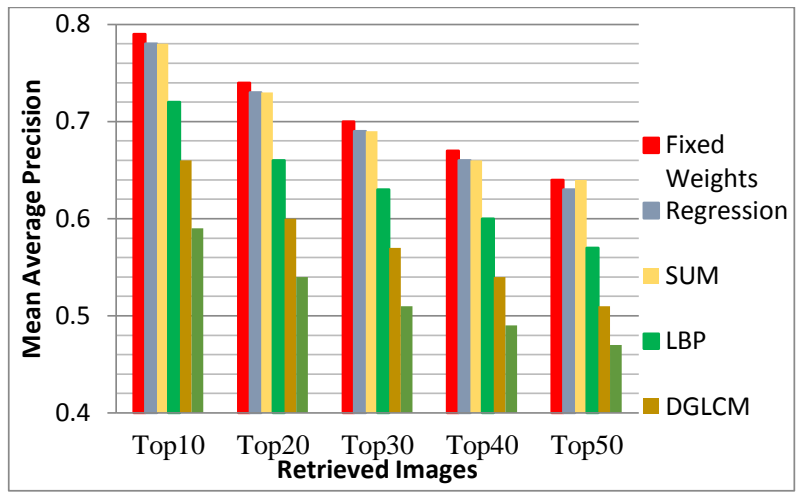

Fig.11. The Fusion Versions using LBP, DGLCM, and DWT for YCbCr images.

Table 8. Comparison of MAP for Top 10 Retrieved Images using WANG database

\begin{tabular}{|c|c|}
\hline Method & MAP \\
\hline Proposed algorithm & 0.79 \\
\hline$[21]$ & 0.66 \\
\hline$[25]$ & 0.76 \\
\hline$[26]$ & 0.66 \\
\hline
\end{tabular}

\section{CONCLUSION}

This paper presented an algorithm for CBIR that addresses the semantic gap challenge by integrating colour and texture visual information called a data-level fusion to represent images using different global features from spatial (GLCM and LBP) and frequency domain (DWT). The experimental study results proved that integrated GLCM, LBP, and DWT from $\mathrm{Y}, \mathrm{Cb}$, and $\mathrm{Cr}$ channels can support in further discriminating images in different classes that could involve common objects. In addition, the algorithm applied a score-level fusion on similarity values (evidences) obtained from mentioned features after normalization. Consequently, the accuracy of image retrieval was highly affected. This seeming that the insufficiency of evidences can be complemented by fusing.

In the future work, the algorithm can be tested on local features and LBP feature using different number of neighbours $(N)$ and radius $(R)$ because the feature performance was the best compared to GLCM and DWT features. In addition the algorithm can be evaluated on larger databases such as Caltech256.

\section{REFERENCES}

[1] A. W. Smeulders et al., "Content-based image retrieval at the end of the early years, "Pattern Analysis and Machine Intelligence, IEEE Transactions on, 22(12), pp. 13491380, 2000.

[2] R. Datta, D. Joshi, J. Li \& J. Z. Wang, J. Z., "Image retrieval: Ideas, influences, and trends of the new age," ACM Computing Surveys (CSUR), 40(2), p. 5E, 2008.

[3] Haralick, R. M., Shanmugam, K. \& Dinstein, I. H. , "Textural features for image classification," IEEE Transactions on Systems, Man and Cybernetics, SMC3(6), pp. 610-621, 1973.

[4] J. Prabhu and J. S. Kumar, "Wavelet based content based image retrieval using color and texture feature extraction by gray level co ocurence matrix and color coocurence matrix," Journal of Computer Science, Science Publication 10 (1), p.15-22, 2014.

[5] N. Bagri and P. K. Johari, P., "A Comparative Study on Feature Extraction using Texture and Shape for Content Based Image Retrieval," International Journal of Advanced Science and Technology Vol.80, ISSN: 20054238, pp.41-52.P, 2015.

[6] M. Benco, R. Hudec, P. Kamencay, M. Zachariasova, and S. Matuska, "An Advanced Approach to Extraction Color Texture Features Based on GLCM," International Journal of Advanced Robotic Systems, ISSN 1729-8806, 2014.

[7] N. Puviarasan, R. Bhavani and A. Vasanthi, "mage Retrieval Using Combination of Texture and Shape Features," International Journal of Advanced Research in Computer and Communication Engineering, Vol. 3, Issue 3, 2014.

[8] R. Putri, H. Prabawa and Y. Wihardi, "Color and texture features extraction on content-based image retrieval," $3 \mathrm{rd}$ International Conference on Science in Information Technology (ICSITech), IEEE, 2017.

[9] M. V. Lande, P. Bhanodiya and P. Jain, "An effective content-based image retrieval using color, texture and shape feature," In Intelligent Computing, Networking, and Informatics(pp. 1163-1170). Springer, New Delhi.Horvath, 2014.

[10] R. Kaur and I. Singh, "Image Retrieval System Using Improved Local Binary Patterns and GLCM Matrices," Imperial Journal of Interdisciplinary Research, 3(6), 2017.

[11] Ahonen, T., Hadid, A. and Pietikainen, M., 2006. Face description with local binary patterns: Application to face recognition. IEEE Transactions on Pattern Analysis \& Machine Intelligence, (12), pp.2037-2041.

[12] P. Liu, J. M. Guo, K. Chamnongthai, and H. Prasetyo, "Fusion of color histogram and LBP-based features for texture image retrieval and classification," Information Sciences, 390, pp.95-111, 2017.

[13] C. Singh, E. Walia, and K. P. Kaur, "Color texture description with novel local binary patterns for effective image retrieval," Pattern recognition, 76, pp.50-68, 2018. 
[14] J. Z. Wang, Li,J. and G. Wiederhold, "SIMPLIcity: Semantics-sensitive integrated matching for picture libraries," Pattern Analysis and Machine Intelligence, IEEE Transactions on, 23(9), pp. 947-963, 2001.

[15] M. Petrou and C. Petrou, "Image processing: the fundamentals," Book, John Wiley $\backslash \&$ Sons, 2010.

[16] R. Hall, "Illumination and color in computer generated imagery," Springer Science \& Business Media, 2012.

[17] H. Al-Jubouri, "Multi evidence fusion scheme for contentbased image retrieval by clustering localised colour and texture features," Doctoral thesis, University of Buckingham, 2015.

[18] A. Eleyan and H. Demirel, "Co-occurrence matrix and its statistical features as a new approach for face recognition," Turk J Elec Eng \\& Comp Sci, 19(1), pp. 97-107, 2011.

[19] E. A. Fox and J., A. Shaw, "Combination of Multiple Searches," NIST SPECIAL PUBLICATION SP, pp. 243246, 1994.

[20] J. Lee, "Analyses of Multiple Evidence Combination," New York, NY, USA, Proceedings of the 20th annual international ACM SIGIR conference on Research and development in information retrieval, pp. 267-276, 1997.

[21] L. Feng, J. Wu, S. Liu and H. Zhang, "Global correlation descriptor: a novel image representation for image retrieval," Journal of Visual Communication and Image Representation, Vol.33, pp.104-114, 2015.

[22] T. Ojala, M. Pietikainen, and D. Harwood, "Performance evaluation of texture measures with classification based on Kullback discrimination of distributions," Proceedings of the 12th International Conference on Pattern Recognition Computer Vision $\backslash \&$ Image Processing, pp. 582-585, 1994.

[23] T. Ojala, M. Pietikainen, and T. Maenpaa, "Multiresolution gray-scale and rotation invariant texture classification with local binary patterns," Pattern Analysis and Machine Intelligence, IEEE Transactions on, 24(7), pp. 971-987, 2002.

[24] A. Field, "Discovery statistics using SPSS", London, UK: SAGE Publications, 2006.

[25] M. K. Kundu, M. Chowdhury, and S.R. Bulò, "A graphbased relevance feedback mechanism in content-based image retrieval", Knowledge-Based Systems, Vol.73, pp.254-264, 2015 .

[26] L. Feng, J. Wu, S. Liu and H. Zhang. "Global correlation descriptor: a novel image representation for image retrieval", Journal of Visual Communication and Image Representation, Vol.33, pp.104-114, 2015.

\section{Author's Profile}

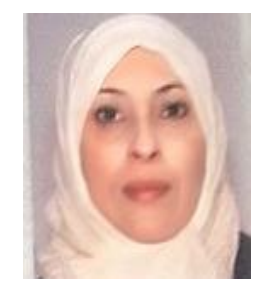

Hanan A. Al-Jubouri (Iraq, Baghdad, 1971). B.Sc. of Computer Science, Technology University, Baghdad, Iraq, 1994. MSc. of Computer Science, Technology University, Baghdad, Iraq, 2001. PhD. of Computer Sciencel Information System, Buckingham University, Buckingham, UK, 2015.

She worked as a PROGRAMMER and then ASSTANT LECTURER in Mustansiriyah University. Currently, she is working as a LECTURER in Computer Engineering Department/ Mustansiriyah University/ Iraq. Research interests are mainly Content-Based Image Retrieval and Data mining.

How to cite this paper: Hanan A. Al-Jubouri, " Integration Colour and Texture Features for Content-based Image Retrieval", International Journal of Modern Education and Computer Science(IJMECS), Vol.12, No.2, pp. 10-18, 2020.DOI: 10.5815/ijmecs.2020.02.02 